\title{
Possibilities and limitations of direct detection of protein charges by means of an immunological field-effect transistor
}

\author{
R.B.M. Schasfoort ${ }^{2}$ and P. Bergveld * \\ Department of Electrical Engineering. University of Twente, P.O. Box 217, 7500 AE Enschede (The Netherlands) \\ R.P.H. Kooyman and J. Greve \\ Department of Applied Physics, University of Twente, P.O. Box 217, 7500 AE Enschede (The Netherlands)
}

(Received 1st February 1990)

\begin{abstract}
An outline of the requirements for the construction of an immunological field-effect transistor (ImmunoFET) which should operate on the direct potentiometric sensing of protein charges is given. Selectivity of the ImmunoFET can be obtained by immobilizing antibodies on the gate area of the ISFET, enhancing the surface affinity to the corresponding antigens over other molecules in the solution. A theoretical approach is given based on the Donnan equilibrium description, which provides an insight into the potential and ion distribution in the protein layer on the ImmunoFET. It is shown that the Donnan potential and the internal pH shift, induced by the protein charges, compensate each other to a great extent. If the ISFET shows Nernstian behaviour, it is concluded that a direct detection of protein charge is impossible. In order to construct an ImmunoFET, a reference FET (REFET) or ISFET with low sensitivity would satisfy the detection of the partially compensated Donnan potential in the presence of an adsorbed protein layer. However, the application of such an ImmunoFET is limited to samples with low ionic strength.
\end{abstract}

Keywords: Proteins; Immunological field-effect transistor

The determination of proteins by measuring an intrinsic property such as charge density, molecular weight, three-dimensional structure, dielectric constant or refractive index of the protein itself is an attractive possibility for constructing an immunological biosensor [1]. The conditions required for the direct detection of charge alteration due to an immune reaction will be considered. In the literature some methods have been described for the direct detection of charge densities of proteins by means of biosensor techniques. These can be

\footnotetext{
Present address: Department of Biotechnology, Applied Research Institute (TNO), P.O. Box 108, 3700 AC Zeist, The Netherlands.
}

classified under three distinct headings areas: lipid-protein interaction either at black lipid bilayer membranes (BLM) or at Langmuir-Blodgett (LB) monolayers; ion-selective electrode-related techniques and direct potentiometric sensing of the charges of proteins on solid-state electrodes or devices. There are many papers related to these subjects. In this introduction, only some key papers will be considered which are related to the last area.

In 1975 a very simple immuno electrode was reported [2]. Direct detection of protein charge was accomplished with a PVC-coated platinum wire. The potential of the electrode with respect to an $\mathrm{Ag} / \mathrm{AgCl}$ electrode changed owing to adsorp- 
tion of charged macromolecules. Although the system reported was not based on an immunochemical reaction, the finding of a potentiometric response in a concanavalin A-polysaccharide model system stimulated further investigations in this field.

A related immunoelectrode was described by Yamamoto et al. [3]. Direct potentiometric sensing of the antigen hCG with an antibody (anti-hCG)sensitized titanium wire resulted in $5 \mathrm{mV}$ shifts with respect to a saturated calomel electrode. Moreover, the potential of an antigen-sensitized electrode resulted in a reversed signal on addition of anti-hCG to the solution. Detection of $10^{-7} \mathrm{M}$ hCG was possible. The change in potential was explained by a simple charge-transfer model.

Subsequently Schenck [4] proposed the detection of an immunological reaction by means of an immunological field-effect transistor (ImmunoFET). He suggested employing the FET with, on the gate region, a layer of antibody specific to a particular antigen. Replacement of the electrolyte solution with another electrolyte solution containing the antigen should alter the charge of the protein surface layer due to the antigen-antibody reaction, thus affecting the charge concentration in the inversion layer of the transistor. The corresponding change in the drain current would then provide a measure of the antigenic protein concentration in the replacement solution.

Many research groups have tried to realize the proposed concept of Schenck, but the results obtained are meager. Moreover, Janata [5] reported that it is impossible to construct an ImmunoFET without having an ideal polarized solution-insulator interface. He qualified all of his earlier results as artifacts.

However, recently Gotoh et al. [6] published results obtained with an ImmunoFET. A 2-mV shift was detected with a human serum albumincontaining polyvinylbutyral membrane deposited on an ISFET after reaction with its antibody. A linear relationship was obtained with an antibody concentration range in the sample solution of $10^{5}-10^{-7} \mathrm{M}$.

It seems that experimental results obtained with direct detection of proteins on solid-state electrodes or similar devices are so far limited to second-order effects $[7,8]$. However, a real theoretical explanation is absent. The purpose of this paper is to consider in detail the FET as a direct protein sensor. In particular, the theoretical implications and practical requirements for constructing an ImmunoFET are considered in detail.

THE ISFET AS A DIRECT PROTEIN CHARGE MEASURING DEVICE

The amino acids in a polypeptide chain have specific $\mathrm{p} K$ values. Therefore, the charge of a protein depends mainly on the amino acid composition and $\mathrm{pH}$. Each protein has its characteristic isoelectric point (the point where the net charge is zero) and charge change per $\mathrm{pH}$ unit (which depends on composition and the titration curve). An elementary way to describe charge changes if two proteins combine, as will be the case in an immunological reaction, is as follows [9].

Assume that the immunoglobulins each carry a charge $X_{1}$, the polarity and magnitude of which depend on the $\mathrm{pH}$ of the solution. After the reaction with the corresponding antigens with charges $X_{2}$, there is a newly formed antibody-antigen complex with charge $X_{3}$ :

$\mathrm{Ab}^{X_{1}}+\mathrm{Ag}^{X_{2}} \stackrel{K}{\leftrightharpoons}[\mathrm{AbAg}]^{X_{3}}$

Ideally, the charge change from $X_{1}$ to $X_{3}$ should be detectable by a charge measuring device, such as an ISFET. It is therefore necessary to obtain more insight into the mechanism of ISFET charge detection. Basically, an ISFET detects charge in a capacitative way. The solution is grounded by a reference electrode and the electric field in the FET gate insulator depends on, among other things, the net surface charge at the electrolyte-insulator interface. A charge density change at the insulator surface will result in an equal change in charge density of opposite sign in the inversion layer of the FET.

The question arises as to whether the net charge of a protein at the electrolyte-insulator interface will be detectable in this way, making possible the determination of protein concentrations. Selectivity of such a device can then be obtained by 
immobilizing antibodies on the gate area of the ISFET, resulting in specific reaction with antigens. This so-called immunological field-effect transistor (ImmunoFET) enhances the surface affinity to antigens over other molecules in the solution and the signal measured by the ImmunoFET would be mostly due to the reaction with that particular antigen. It is obvious that the charge distribution in the immediate vicinity of the interface plays a decisive role in transferring an immunological signal to the ImmunoFET.

\section{ELECTROSTATIC EFFECTS AT THE ISFET SURFACE}

The charge redistribution around immobilized proteins at the insulator-solution interface can be described by the double-layer theory [10]. Here only a qualitative treatment will be given of the electrostatic influence of protein charges which penetrate in the diffuse Gouy-Chapman layer at the insulator-solution interface.

On adsorption the diffuse layer of counter ions around the protein charges may overlap with the diffuse layer of the electrolyte-insulator interface as represented in Fig. 1. The thickness of diffusecharged layers is described by the Debye theory [11] and defined by the distance where the electrostatic field has dropped to 1 /e of its initial value:

$\kappa^{-1}=\left(\frac{\epsilon_{0} \epsilon k T}{2 q^{2} I}\right)^{1 / 2}$

with $\kappa^{-1}$ is the Debye length, $q$ the elementary charge, $k$ Boltzmann's constant, $T$ absolute temperature, $\epsilon_{0}$ the permittivity of vacuum, $\epsilon$ the dielectric constant and $I=1 / 2 \sum c_{i} z_{i}^{2}$ represents the ionic strength, while $c_{i}$ is the concentration of ion $i$ with valency $z$ (for a 1-1 salt $I$ can be replaced by $c$ ).

It can be seen from Eqn. 1 that the Debye length is strongly dependent on the ionic strength of the solution; more precisely, the Debye length is inversely proportional to the square root of the ionic strength. Therefore, one can expect that the chance of overlapping of the double layers of the substrate-solution interface and the adsorbed proteins can be substantial only if low electrolyte

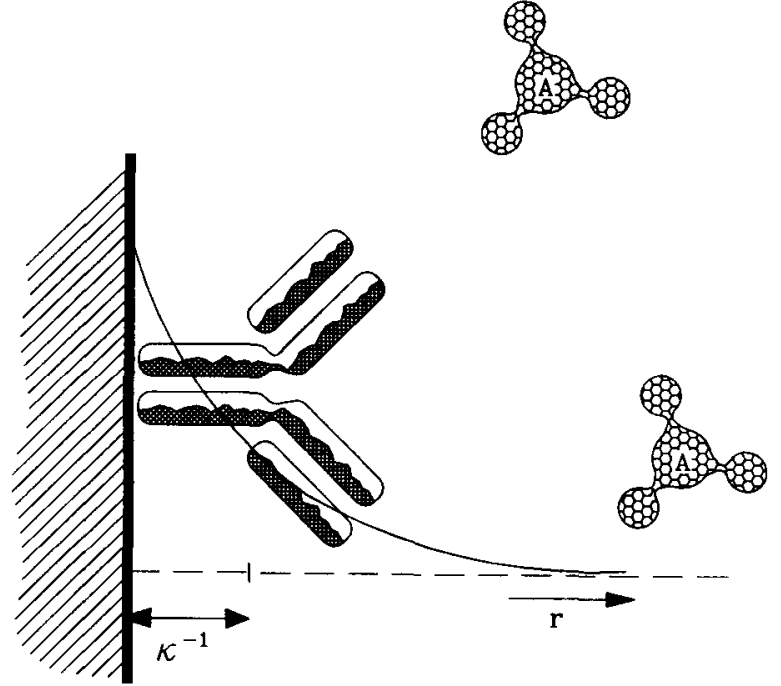

Fig. 1. Schematic representation of the overlapping double layer of the electrolyte-solution interface with an adsorbed antibody molecule. Only the diffuse part of the double layer of the electrolyte-solution interface is shown. The line gives the potential in arbitrary units as a function of the distance $r$ from the surface. $\kappa^{-1}$ is the Debye length and was calculated for 10 $\mathrm{mM} \mathrm{KCl}$ solution to be ca. $3 \mathrm{~nm}$. A represent antigens present in the solution.

concentrations are used, owing to the dimensions of the proteins.

In a physiological salt solution the Debye length is limited to ca. $0.8 \mathrm{~nm}$. It is obvious that only charge density changes that occur within the order of a Debye length of the ISFET surface can be detected. With macromolecules, such as proteins, the dimensions are much larger (ca. $10 \mathrm{~nm}$ ) than those of the double layer of the electrolyte-insulator interface. This means that in such a case most of the protein charge will be at a distance greater than the Debye length from the surface.

If, moreover, on top of a monolayer of antibody molecules a second layer of antigens is coupled, it is obvious that the chance of overlap of the diffuse layers of antigens with the electrolyte-substrate interface will decrease even more. At high ionic strength the additional charges of the antigens are nearly always located far outside the diffuse layer at the ISFET surface and pure electrostatic detection of these antigenic charges will therefore be impossible. Janata and Huber [9] 
estimated a $10-\mathrm{mV}$ signal change, for a "best case" situation of nearly complete antibody coverage, coupled with highly charged antigens at very low ionic strength.

In addition to the purely electrostatic effects (overlap of the diffuse layers), one must also take into consideration the implication that the diffuse layers have a different chemical composition to the bulk solution, i.e., the $\mathrm{pH}$ in the diffuse layer deviates from that of the bulk solution. With proteins this complicates the description because the protein charge is a function of $\mathrm{pH}$. Davies and Rideal [11] calculated that the $\mathrm{pH}$ near a charged interface depends on the potential $\phi$ in the diffuse layer according to

$\mathrm{pH}_{\mathrm{i}}=\mathrm{pH}_{\mathrm{s}}+\frac{F \phi}{2.3 \mathrm{R} \mathrm{T}}$

where $\mathrm{pH}_{\mathrm{i}}$ is the $\mathrm{pH}$ at the interface, $\mathrm{pH}_{\mathrm{s}}$ is the $\mathrm{pH}$ in the bulk solution and $R, T$ and $F$ have their usual meanings. A typical value of $200 \mathrm{mV}$ near the electrolyte-insulator interface would result in $\mathrm{pH}$ changes of 3-4 units.

It must be concluded that the effects that an adsorbed protein layer has on the potential redistribution at a charged interface are interrelated in a complex way to the interfacial charge, the ionic strength of the solution, the $\mathrm{pH}$ of the solution, the effective distance of the protein layer from the surface, the chemically induced alterations, etc.

Independent of the double-layer description, including the protein-related chemical interaction as mentioned above, Donnan described the theory of potential generation at the membrane-electrolyte interface as a function of the charged species in the electrolyte. This theory can also be applied to electrolyte-membrane interfaces where the membrane contains fixed charges such as immobilized proteins. This is described below in order to model the effect of the protein layer on the actual measurement with an underlying ISFET.

ELECTROCHEMICAL EFFECTS AT THE ISFET SURFACE

\section{Description of the Donnan equilibrium}

In this section the electrochemical effect of amphoteric macromolecules adsorbed to the

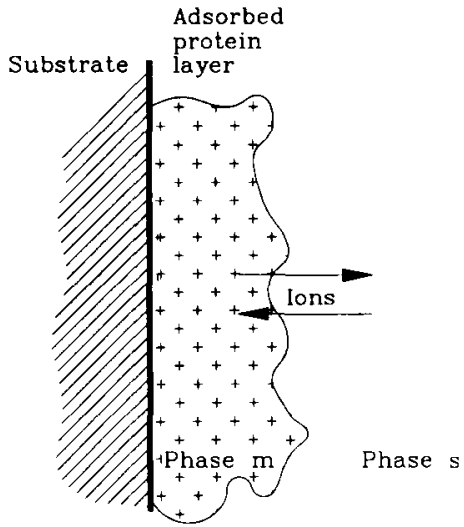

Fig. 2. The considered system for the description of the Donnan potential. A protein membrane (phase $m$ ) with fixed charge density $c_{\mathrm{x}}$ in equilibrium with the bulk solution (phase s). Small ions of the 1-1 salt are assumed to diffuse freely through the system.

ISFET is described from a thermodynamic point of view. Consider a system which consists of two phases (Fig. 2) where phase s contains a solution with only a 1-1 salt, having ions $\mathrm{A}^{+}$and $\mathrm{B}^{-}$, and phase $\mathrm{m}$ contains charged macromolecules deposited on a substrate and considered as a membrane with fixed charges.

Assume that the small ions can diffuse freely through phase $s$ in addition to phase $\mathrm{m}$, in contrast to the fixed charges in the membrane. As a result of the presence of the fixed charges of the macromolecules, there is a difference in the ion concentration between phases $\mathrm{s}$ and $\mathrm{m}$, which at equilibrium must satisfy the equality of electrochemical potentials [10]:

$$
\begin{aligned}
\mu_{0 \mathrm{~A}^{+}}+R T \ln a_{\mathrm{A}^{+}, \mathrm{s}}+z F \phi_{\mathrm{s}}= & \mu_{0 \mathrm{~A}^{+}}+R T \ln a_{\mathrm{A}^{+}, \mathrm{m}} \\
& +z F \phi_{\mathrm{m}} \\
\mu_{\mathrm{OB}^{-}}+R T \ln a_{\mathrm{R}^{-}, \mathrm{s}}+z F \phi_{\mathrm{s}}= & \mu_{0 \mathrm{~B}^{-}}+R T \ln a_{\mathrm{B}^{-}, \mathrm{m}} \\
& +z F \phi_{\mathrm{m}}
\end{aligned}
$$

where $\mu_{0 \mathrm{i}}$ is the electrochemical standard potential of ion $\mathrm{A}^{+}$or $\mathrm{B}^{-}, R, T$ and $F$ have their usual meanings, $a_{\mathrm{i}}$ is the activity of ion $\mathrm{A}^{+}$or $\mathrm{B}^{-}, \phi_{\mathrm{s}}$ and $\phi_{\mathrm{m}}$ are the electrical potentials in phase $s$ and $\mathrm{m}$, respectively, and $z$ is the valency of the ion (for a 1-1 salt, $z=1$ or -1 ).

Rearranging Eqn. 3 gives

$$
\begin{aligned}
\phi_{\mathrm{m}}-\phi_{\mathrm{s}} & =R T / F \ln a_{\mathrm{A}^{+}, \mathrm{s}} / a_{\mathrm{A}^{+}, \mathrm{m}} \\
& =R T / F \ln a_{\mathrm{B}^{-}, \mathrm{m}} / a_{\mathrm{B}^{-}, \mathrm{s}}
\end{aligned}
$$


The Donnan ratio $\left(r_{\mathrm{d}}\right)$ is given by

$r_{\mathrm{d}}=a_{\mathrm{A}^{+}, \mathrm{s}} / a_{\mathrm{A}^{+}, \mathrm{m}}=a_{\mathrm{B}^{-}, \mathrm{m}} / a_{\mathrm{B}^{-}, \mathrm{s}}$

At equilibrium all ions present in the sample solution, including $\mathrm{H}_{3} \mathrm{O}^{+}$and $\mathrm{OH}^{-}$, are distributed according to this Donnan ratio. Hence the $\mathrm{pH}$ in phase $m$ is different from that in phase $s$, according to

$\mathrm{pH}_{\mathrm{m}}-\mathrm{pH}_{\mathrm{s}}=\log r_{\mathrm{d}}$

Note that for Eqn. 2 this implies that the potential term $\phi$ can be replaced by the potential difference $\left(\phi_{\mathrm{m}}-\phi_{\mathrm{s}}\right)$ between the membrane and the solution $\left(\mathrm{pH}_{\mathrm{i}}\right.$ will be $\left.\mathrm{pH}_{\mathrm{m}}\right)$.

Further, the bulk electroneutrality condition must always be fulfilled in the system. The electroneutrality equations are

$a_{\mathrm{A}^{+}, \mathrm{s}}-a_{\mathrm{B}^{-}, \mathrm{s}}=0$

and

$a_{\mathrm{A}^{+}, \mathrm{m}}-a_{\mathrm{B}^{-}, \mathrm{m}}+c_{\mathrm{x}}=0$

where $c_{\mathrm{x}}$ represents the effective fixed charge density in phase $\mathrm{m}$. Combination of Eqns. 4 and 7 results in

$a_{\mathrm{A}^{+}, \mathrm{m}}=1 / 2\left(\sqrt{4 a_{\mathrm{s}}^{2}+c_{\mathrm{x}}^{2}}-c_{\mathrm{x}}\right)$

and

$a_{\mathrm{B}^{-}, \mathrm{m}}=1 / 2\left(\sqrt{4 a_{\mathrm{s}}^{2}+c_{\mathrm{x}}^{2}}+c_{\mathrm{x}}\right)$

where $a_{\mathrm{s}}$ is the salt activity in the sample solution and equals $a_{\mathrm{A}^{+}, \mathrm{s}}$ and $a_{\mathrm{B}^{-}, s^{-}}$. It should be mentioned that the overall partition coefficient is assumed to be 1 . This means that in an uncharged membrane the salt activity in the membrane is considered to be equal to that in the bulk solution. The potential difference between phases $m$ and $s$ is

$\Delta \phi_{\mathrm{d}}=\phi_{\mathrm{m}}-\phi_{\mathrm{s}}=R T / F \ln \frac{\left(\sqrt{4 a_{\mathrm{s}}^{2}+c_{\mathrm{x}}^{2}}+c_{\mathrm{x}}\right)}{2 a_{\mathrm{s}}}$

where $\Delta \phi_{d}$ is the Donnan potential between membrane and solution. The theoretical description given here is sufficient to understand the influence of the Donnan effect on the system considered below and is represented graphically by curves a in Fig. 3 for different values of $c_{\mathrm{x}}$.

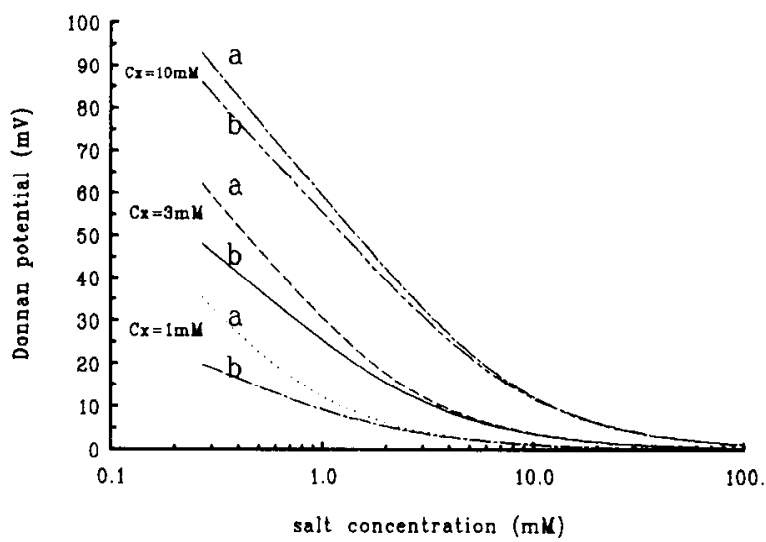

Fig. 3. Donnan potential as a function of the salt concentration for three fixed charge densities $(1,3$ and $10 \mathrm{mM})$ of the protein layer (lines a, Eqn. 9). Lines b were calculated numerically by using Eqns. 9 and 15.

The Donnan effect applied to a protein membrane deposited on an ISFET

The Donnan effect affects both the Donnan potential and the $\mathrm{pH}$ difference between the two phases as described in the previous section. Thus, in order to measure the presence of proteins in a membrane as a Donnan potential $\Delta \phi_{\mathrm{d}}$ by means of a $\mathrm{pH}$ measuring device such as the ISFET, the influence of the Donnan effect on the potential generation and on the internal membrane $\mathrm{pH}$ has to be considered. Both will affect the ISFET drain current. Assuming that the response of a bare ISFET to the $\mathrm{pH}$ of the solution is

$V_{\mathrm{gs}}=\alpha R T / F \ln a_{\mathbf{H}^{+}, \mathrm{s}}+$ constant

where $V_{g s}$ is the gate-source voltage of an ISFET connected to a source drain follower [12]. The constant term reflects a combined potential including the reference electrode potential and other constants and $\alpha$ is the sensitivity factor of the device, obtained by calibrating the ISFET.

If a protein layer is deposited on the ISFET, the Donnan adapted $a_{\mathrm{H}^{+}, \mathrm{m}}$ and the Donnan potential $\left(\Delta \phi_{d}\right)$ are now measured. The gate-source voltage of the protein coated ISFE'I is therefore

$V_{\mathrm{gs}}^{\prime}=\alpha R T / F \ln a_{\mathrm{H}^{+}, \mathrm{m}}+\Delta \phi_{\mathrm{d}}+$ constant

The deviation of the gate-source voltage due to the presence of the protein is thus

$V_{\mathrm{gs}}^{\prime}-V_{\mathrm{gs}}=\alpha R T / F \ln \left(a_{\mathrm{H}^{+}, \mathrm{m}} / a_{\mathrm{H}^{+}, \mathrm{s}}\right)+\Delta \phi_{\mathrm{d}}$ 
Combining this with Eqn. 4 results in

$V_{\mathrm{gs}}^{\prime}-V_{\mathrm{gs}}=(1-\alpha) \Delta \phi_{\mathrm{d}}$

If the ISFET shows a Nernstian behaviour ( $\alpha$ $=1$ ), it can be concluded that the gate-source voltage of the ISFET is not influenced by proteins adsorbed in the membrane on the gate of an ISFET. The Donnan potential difference is then fully compensated by the internal membrane $\mathrm{pH}$. If the response of the ISFET deviates from the Nernstian response $(\alpha<1)$ then a shift in the gate-source voltage can be expected on protein adsorption. In general, partial compensation of the Donnan potential will always take place, provided that the conditions are fulfilled such that the Donnan effect manifests itself.

\section{Implication of the amphoteric character of pro-} teins on the Donnan potential

In addition to the described compensation effect, the amphoteric character of the protein layer complicates a further detailed description. The charge density of the protein membrane changes indirectly owing to the change in membrane $\mathrm{pH}$. Thus, in Eqn. 9 not a constant value of the charge density but a pH-dependent value must be inserted. Assume the effective charge density $\left(c_{x}\right)$ of the protein membrane to be a function of $\mathrm{pH}$ according to

$c_{\mathrm{x}}=c_{\mathrm{m}} S_{\mathrm{i}} c_{\mathrm{i}}\left(\mathrm{p} I_{\mathrm{i}}-\mathrm{pH}_{\mathrm{m}}\right)$

where $S_{\mathrm{i}}$ is a factor which depends on the slope of the titration curve of the protein, $c_{i}$ is the protein concentration in the membrane, $c_{\mathrm{m}}$ is a constant with dimensions of $c_{\mathrm{x}}, \mathrm{p} I_{\mathrm{i}}$ is the isoelectric point of the protein and $\mathrm{pH}_{\mathrm{m}}$ is the internal membrane $\mathrm{pH}$. Equation 14 is valid in a region of $\pm 2-3 \mathrm{pH}$ units around the isoelectric point of the protein [13].

Substituting Eqns. 6 and 4 results in

$c_{\mathrm{x}}=c_{\mathrm{m}} S_{\mathrm{i}} c_{\mathrm{i}}\left(\mathrm{p} I_{\mathrm{i}}-\mathrm{pH}_{\mathrm{s}}-\frac{F \Delta \phi_{\mathrm{d}}}{2.3 R T}\right)$

Applying Eqn. 15 for a positive value of $c_{\mathrm{x}}, \Delta \phi_{\mathrm{d}}$ will be positive and become more positive with decreasing salt concentration, which means that the value of $c_{x}$ will decrease. On the other hand, if $c_{\mathrm{x}}$ is negative, a decreasing salt concentration will result in a less negative value of $c_{\mathrm{x}}$. Summarizing, the charge density will always shift towards zero, which means that $\mathrm{pH}_{\mathrm{m}}$ will always shift to $\mathrm{p} I_{\mathrm{i}}$. In fact, the Donnan phenomenon balances the $\mathrm{pH}$ and the effective membrane charge density. The effect of the implication of the amphoteric character of the proteins can be calculated numerically according to Eqns. 9 and 15 and is represented by curves $b$ in Fig. 3.

From Fig. 3, it can be concluded that, although the amphoteric character of the proteins complicates the calculations for the Donnan potential, it does not influence the assertion of the compensation effect described in the previous section.

\section{Conclusions}

An outline of the possibilities and limitations of an ISFET as a protein sensor has been given. An attempt was also made to establish the requirements for the construction of an ImmunoFET which should operate on the direct potentiometric sensing of protein charges. With monolayer adsorption, the protein must fit into the double layer at the electrolyte-insulator interface and the dimensions of the protein must be smaller than the Debye length. The ionic strength of the solution is the important parameter in controlling the Debye length in a solution and it should be as low as possible. However, the exact influence of overlapping double layers of the electrolyte-solution interface and the protein charges on the ISFET response is difficult to describe.

If the protein layer is considered as a membrane deposited on an ISFET, the electrostatic effects can be described by the thermodynamic Donnan equilibrium description. The Donnan equilibrium description provides an insight into the potential and ion distribution in the layer on the ISFET. It was shown that the Donnan potential and the internal membrane $\mathrm{pH}$ shift, induced by the protein charges, may compensate each other. The amphoteric character of proteins does not influence this compensation effect. It causes a decrease in effective charge density because of a $\mathrm{pH}$ shift in the protein layer towards the isoelectric point of the protein, resulting in a reduced Donnan potential. 
A relationship exists between the electrostatic effect at a membrane surface, which can be described by the double-layer theory, and the electrochemical description of potential generation in a charged membrane based on the Donnan equilibrium effect. This was described by Ohshima and Ohki [14], who solved the Poisson-Boltzmann equation at the outside and inside of a charged membrane. This model smoothly connects the electrostatic and electrochemical potential values. Therefore, the electrostatic and electrochemical effects described in this paper are strongly interrelated.

If the ISFET as a $\mathrm{pH}$ sensor shows Nernstian behaviour $(\alpha=1)$, it should be concluded that a direct detection of protein charge is impossible. However, if it were possible to construct a modified ISFET, which measures the electrical potential of the solution only without exhibiting sensitivity for any ion present in the sample solution (a so-called reference FET (REFET) [15]; $\alpha=0$ ), the detection of the Donnan potential generated by the fixed charges of the proteins would become feasible. Even a well characterized ISFET with known $\alpha<1$ would satisfy the detection of the $\mathrm{pH}$-compensated Donnan potential resulting from the presence of an adsorbed immunological laycr.
However, in all instances the application of such an ImmunoFET is limited to samples with low ionic strength.

\section{REFERENCES}

1 M. Aizawa, Philos. Trans. R. Soc. London, 316 (1987) 121.

2 J. Janata, J. Am. Chem. Soc., 97 (1975) 10.

3 N. Yamamoto, Y. Nagasawa, M. Sawai, T. Sudo and H. Tsubomura, J. Immunol. Methods, 22 (1978) 309.

4 J.F. Schenck, U.S. Pat., 4238 757, 1980.

5 J. Janata, in J.-L. Aucouturier et al (Eds.), Proceedings of the 2nd International Meeting on Chemical Sensors, Bordeaux, 1986, University of Bordeaux, Bordeaux, 1986, pp. 25-31.

6 M. Gotoh, E. Tamiya and I. Karube, J. Membrane Sci., 41 (1989) 291.

7 S. Collins and J. Janata, Anal. Chim. Acta, 136 (1982) 93.

8 R.B.M. Schasfoort, G. Streekstra, R.P.H. Kooyman, P. Bergveld and J. Greve, Sensors Actuators, 18 (1989) 119.

9 J. Janata and R.J. Huber, in H. Freiser (Ed.), Analytical Chemistry, Vol. 22, Plenum, New York, 1980, p. 1156.

10 W.J. Moore, Physical Chemistry, Longman, London, 1976.

11 J.T. Davies and E.K. Rideal, Interfacial Phenomena, Academic, London, 1963.

12 P. Bergveld, Sensors Actuators, 1 (1981) 17.

13 W. Norde, Adv. Colloid Interface Sci., 25 (1986) 267.

14 H. Oshima and S. Ohki, Biophys. J., 147 (1985) 673.

15 A. Van den Berg, P. Bergveld, D.N. Reinhoudt and E.J.R. Sudhölter, Sensors Actuators, 8 (1985) 129. 